\title{
Applying Tufte's Principles of Information Design to Creating Effective Web Sites
}

Beverly B. Zimmerman, Brigham Young University, beverly_zimmerman@byu.edu

\begin{abstract}
Edward Tufte's general principles of information design can be applied to effective web design. This paper discusses how to use micro/macro design, layering and scparation, small multiples, color and information, integration of words and images to create effective web sites.
\end{abstract}

\section{Keywords}

Information design, document design, web page design, home pages

\section{Introduction}

Information design-the theory and practice of presenting information in a comprehensive, usable, and effective manner-is an evolving field with ever-expanding boundaries. Nowhere is the need to move the boundaries of information design more evident than in designing information for the World Wide Web. Just as the desktop-publishing movement of the $80 \mathrm{~s}$ meant new practices for producing informational documents, so the World Wide Web means new practices for distributing information. With the phenomenally increasing number of web pages appearing on the Internet, web documents may soon become the single most used method for distributing information. Unfortunately, the increased quantity of web sites may not be matched by an increased quality of design at those sites.

A plethora of advice is already being distributed on the World Wide Web itself about how to create effective web pages, but most of this advice takes a "what is hot and what is not" approach. For

Permission to make digital/hard copies of all or part of this material for personal or classroom use is granted without fee provided that the copies are not made or distributed for profit or conmercial advantage, the copyright notice, the title of the publication and its date appear, and notice is given that copyright is by permission of the ACM, Inc. To copy otherwise, to republish, to post on servers or to redistribute to lists, requires specific permission and/or fee.

SIGDOC 97 Snowbird Utah USA

Copyright 1997 ACM 0-89791-861-4/97/10..\$3.50 example, my recent search of the World Wide Web for principles of effective information design yielded many sites with personal perspectives on how to design a web site. These sites included several David Letterman-style "Top Ten" approaches to wcb design: "Top Ten Ways to Make a WWW Flop," "Top Ten Things Not to Do on a Web Page," and "Top Ten Ways to Tell If You Have a Sucky Home Page."

One author suggested that the number one way to improve your home page is to "Be Unique!!! Set your page apart from others!!! Create your own ideas! Go Nuts!" (Glover, http://www.glover.com/ sucky.html, April 9, 1997). On another web page, the author encouraged designers to "create your web site however you want, regardless of what some doofus says is . . ' 'a don't'! Good design is a matter of YOUR personal taste and style, not someone else's! Besides, rules are meant to be broken. I should know . . . I've broken a few of my own!" [bold in the original] (Glover, http://wwwv.glover. com/improve.html, October 11, 1996).

Unfortunately, the quality of web page design varies widely according to each author's skill, experience, and understanding of design principles. Thus, it isn't surprising that one popular web site is titled, "Useless Pages: An Encyclopedia of Links to Terrible Web Sites." Clearly, many web page designers would benefit from an understanding of basic principles of information design.

\section{Objectives of This Paper}

In contrast to the "create your web site however you want" philosophy, Edward Tufte argues in his book Envisioning Information that principles of information design "are universal-like mathematics - and are not tied to unique features of a particular language or culture" $(1990$, p. 10$)$. If he is right, principles of information design aren't unique to a specific medium and can be applied, not only to printed pages, but also to web pages. In this paper, I will use Tufte's information design principles to look at web page design. First, I will explain Tufte's general principles of information design. Then I will show how these general principles underlie many of the practical suggestions given by authors of recent books on how to create web pages. Finally, I will use these principles to evaluate web pages at two web sites. My purpose is to show that general principles of 
information design can be applied to web documents as well as to written documents and can assist web designers in creating effective web pages.

\section{Tufte's Strategies for Envisioning Information}

Tufte describes his general principles of information design as strategies for enabling readers 10 "envision" information-that is, to understand, document. and communicate knowledge. Web page designers should be especially interested in strategies that cnable their readers to understand the information on a web page. Tufte argues that the histon: of every communication device--including web pages. we would assume-is "entircly a progress of methods for enhancing density. complexity. dimensionality, and even sometimes beauty" (1990. p. 33). Furthermore, because readers and creators of information must communicate on the twodimensional "flatland" of the page and video sereen, the goal of the information designer is to help readers escape this flatland by working at the "Intersection of image, word. number. [and] ar" (1990. p. 9).

Tufte's benchmark for communicating information is the high-quality map, designed with an organizational overiew and abundant and varied detail. In contrast to the data-rich visual display of a map is what Tufte calls "chartjunk," a data-thin "postcrization" with contcmpt for both information and audience. Posters-with their emphasis on dominant images, large type. and absence of detailare meant to be glanced at briefly and from a distance. whereas maps reveal rich detal and are meant to be read closely (1990, p. 35).

To help readers avoid chartjunk and posterization. this paper will discuss Tufte's most important design principles: micro/macro design, layering and separation. small multiples, color and information. and integration of words and images.

\section{Micro/Macro Design}

Tufte defines micro/macro design as comprising micro details that "cumulate" or mix into larger macro structures or overall patterns (1990. p. 37). He argues that micro/macro design is a critical and efrective principle of information design that applies to every type of data because it enables readers to understand complex content by giving them an overvicw while at the same time presenting immense detail. By properly arranging and repeating detailed and complex information, the designer creates an overall structure or panorama. Thus, as Tufte emphasizes, the micro/macro design strategy is to clarify by adding detail (1990, p. 38). Those who worry about clutter and information overload, miss the point. "/IJt is not how much emptl' spoce there is, but rather how at is used. It is not how much information there is, but rather how effectrely a is organized" [italics in the original] (1990, p. $5(1)$ ). Effective micro/macro designs are "information thick" providing "a rich texture of data. a comparative context, and [an] understanding of complexity revealed with an econony of means" (1990, p. 51).

This principle of creating a unificd micro/macro structure applies to web page design. For example, it underlies statements by Horton, Taylor. Ignacio, and Hoft, coauthors of The lleb lage Design Cookbook. that "[t]he arrangement of elements on the page is the biggest factor in determining what people notice and read-or whether they abandon the page and surf on to someone else's page" (1996, p. 403). Because viewers see the whole page before they consider the details. Horton and coauthors argue. elements of the page must be designed to accomplish an overall goal. Further, they suggest designers improve the viewer's ability to understand the overall design of a page by providing consistent page banners and by using a consistent style for a group of related pages (1996, p. 440$)$.

The idea of micro/macro design also underlies the guidelines that Carol Clark provides in Irorking the I'eb: A Student's (iunde that designers should define the overall purpose. audience. and objectives for a web site before creating it and should design a template to provide a unified look for sccondary pages (1996, p. 122). Furthermore, Tilton. Steadman, and Joncs, coauthors of lieb l'earing. Destgning and Managing an Effective l'eb Sire. suggest principles of micro/macro design when they discuss "big picture/little picture" concepts such as providing the viewer with an overview at the top of the page or entry point. linking back to the oven icw. and using a unifying theme and consistent form across pages (1996. p. 375). Likcwise, Morris and Hinrichs, authors of Web Page Destgn: il Different Multimedia. refer to micro/macro design when they point out that because of cognitive demands, vewers do not focus on information structures such as tables. groupings, and frames unless they first understand the overall purpose of a site $(1996,86)$. The concep) of micro/macro design also underlies their suggestions that designers create a system overview that is always accessible, and that they combat cognitive overload by stating the goal of the site and by providing a central theme or concept to which details may be connected (1996, p 101). Thus, 
Tufte's basic principle of micro/macro is a common theme, although described using different terminology, in current books on web page design.

\section{Layering and Separation}

Tufte defines layering as visually stratifying or ordering data thereby establishing a proper relationship among types of information. The purpose of layering is to create a visual hierarchy, emphasizing more important content and deemphasizing less important content. In addition, Tufte argues, designers should separate layers of information by means of distinctions of tcxture, weight, shape, value, size, or color. Failure to layer and separate information effectively, he warns, results in "jumbled up, blurry, incoherent, [and] chaotic" designs (1990, p. 58).

This principle of layering and separation is also emphasized in a variety of ways in current books on creating web pages. For example, Tilton and coauthors suggest that designers order information using tables of contents, searchable indexes, and What's New buttons that use a reverse chronological ordering (1996, p. 365). In addition, they urge designers to provide viewers with a clear, consistent navigation structure such as "main roads"

(recommended destinations) and "scenic paths" (side trails and divergences) $(1996$, p. 368). One way to prevent screens of scrolling text or myriads of multidirectional links, they suggest, is to organize content into meaningful classifications using keywords or concepts (1996, p. 375).

The concept of layering underlies Clark's suggestion that designers use a visual outline to plan which main categories of information belong on the page and then to organize those categories into a logical structure that can be linked together (1996, p.124). Her encouragement to use space as a design element, to use headings to group information, and to put important elements in the top left and lower right parts of the screen to allow readers to skim the page (1996, p. 128) are also attempts to visually stratify information.

Layering and separation principles underlie suggestions by Horton and coauthors to divide web pages into blocks of information or distinct zones for a particular kind of information using horizontal rules, swashes, and breaks to make navigating and reading web pages more predictable (1996, p. 405). Layering and separation is also the result of Morris and Hinrichs' recommendation to break content into chunks, group like items together, visually organize data, and put the "most important content in the first 300 vertical pixels of a page" $(1996$, p. 62). Layering and separation also underlies their recommendation that designers divide pages into foreground (attention grabbing), background (foundation), and middleground (organization and structure) and keep foreground items such as blinking text, animation, and marquees to a minimum (1996, p. 80).

It is difficult to tell whether these authors are consciously or inadvertently adopting Tufte's general principle of layering and separation. It may be possible that Tufte's principles are intuitive to many designers or that he is describing an accepted and much-used convention used in advertising design. Nevertheless, Tufte's principle underscores some of the advice currently being given to prospective web page designers.

\section{Small Multiples}

Tufte defines his principle of small multiples as using data-thick "slices" of information to offer variations on a major theme. Resembling the frames of a movie, small multiples are repeated or juxtaposed to allow users to-at a glance-compare changes, see differences among objects, or view alternatives (1990, p. 67). For example, interior designers use similar shaped frames to emphasize a series of pictures on a wall. By keeping a data frame consistent, Tufte argues, designers can isolate detail, maintain context, and emphasize changes in the data rather than changes in design (1983, p. 170).

One aspect of this design principle-keeping comparisons "within the reader's view" (Tufte, 1990, p. 76) - is especially difficult in web page design, but applying the principle of small multiples can overcome this problem. For example, Morris and Hinrichs urge designers to break content into manageable chunks or clusters of five to seven items and to repeat icons, symbols, and menus to assist viewers in comprehending information (1996, p. 98). Clark also suggests that designers use multiples in the form of navigational devices, such as small arrows, icons, and links that are repeated throughout a web site (1996, p. 134). Tilton and coauthors suggest that designers use a small repeated motif or corporate logo in addition to repeating a color scheme and page background (1996, p. 369), in order to maintain a consistent design.

\section{Color and Information}

Tufte argues that tying color to information is another elementary and straightforward principle of information design. But, he warns, it isn't easy. "[E]ven putting a good color in a good place is a complex matter. Indeed, so difficult and subtle that avoiding catastrophe becomes the first principle in 
bringing color to information: Above all, do no harm" [italics in the original] (1990, p. 81). Color has several fundamental uses in information design. Tuffe notes. They are "to label (color as noun), to measure (color as quantity), to represent or imitate reality (color as representation), and to enliven or decorate (color as beauty)" [italics in the original] $(1990$, p. 81$)$. In addition, he suggests that strong colors should be used sparingly as spots against a light gray or dull background tone and that designers be aware that light, bright colors mixed with white next to each other distort the visual field (1990, p. 83). Thus, Tufte urges designers to use earth tones and lighter colors of blue, yellow, and gray. Finally, he argues that color can be used to improve the information resolution of a computer screen, first by softening the bright-white background and secondly by defining lines and edges (1990, p. 90).

Use of color is especially important in web page design. Clark urges designers to use color with restraint and to only use colors that are easy on the eyes (1990, p. 129). Morris and Hinrichs suggest using color to show the structure of various layers of information. Using the right background color can be the "masterstroke" of a web site, they say, so designers should use a color scheme that makes reading text easy and also be aware that strong colors or bizarre color combinations can obscure content. In addition, they tell designers to bc prepared for different cultural interpretations of color (1996, p. 92). For example, the color red doesn't communicate a warning in all cultures. Likewise. Horton and coauthors urge designers to use a background color to make the page look interesting. He also warns, however, that tiled, textured, or brightly colored backgrounds may make text illegible (1996. p. 440).

Although these authors of web page design promote the use of color in a medium that provides for much color, they also suggest using color effectively is a difficult matter and that strong colors should bc used sparingly.

\section{Integrating Words and Images}

The principle of integrating words and images lics at the heart of information design. Tufte argucs, because bringing together words and images enables the designer to tell a story. Although words and Images are different devices, he notes, they have a single purpose--to effectively present information-and therefore must be integrated. Scparating text and graphics, even on the same page. can burden the viewer with the task of associating and linking the separate elements (1990, p. 116). On the other hand, when designers integrate text and figures, the reader can readily interact with and understand the information. Furthermore, focusing on content enables the designer to avoid decorative chartjunk. Tufte warns designers that cosiuctic decoration should never be used to make up for lack of content (1990, p. 35).

Authors of books on creating wcb pages also acknowledge that integrating words and images is important in wcb page design. For example, Clark urges designers to seck a balance between visual sensation and text information. Because readers are looking for content. she warns, pages that are simply flashy color aren't satisfying. Designers should usc tcxt. as well as graphical navigation buttons, to assist viewers in navigating a web page (1996, p. 132). Morris and Hinrichs point out that relationships or associations between information is what determines whether a site is a well rounded and useful. They urge designers to keep attention-grabbing text to a minimum and to arrange text using principles similar to those for arranging text on the printed page: namely, to use both uppercase and lowercase letters and to use well-written, minimalistic text and links in the middleground (1996, p. 89). Tilton and coauthors suggest providing context for linked pages by means of explanatory text or visually associating the link with the subject of the document as a whole (1996, p. 344). Horton and coauthors urge designers to provide textual descriptions of graphics by flowing text around graphics. using tables to position text and graphics together, and by combining text and graphics in page banners to orient the viewer to the purpose and content of the page $(1996$, p. 41$)$.

\section{Evaluating the Effectiveness of Web Pages}

Because Tufte's principles of information design underlie so much of other authors' suggestions for creating web pages, his principles regarding micro/macro design, layering and separation, small multiples. color and information, and integration of words and images can be used to cvaluate the effectiveness of web pages. Using 'Tufte's principles. I will now analyze two sample home pages. These pages, created for different purposes and audiences. also differ in their style and design.

Figure 1 shows the home page of Wells Fargo Bank (http://www wellsfargo.com, Junc 9, 1997). Designers have created micro/macro design by using the name of the company and an image of their familiar stagecoach logo to immediately provide the viewer with an overview of the purpose of the web page. In addition, page headers not only provide the reader with recommended navigational links for 
finding details about online banking, personal finance, small business, commercial banking, and international trade, but also inform the viewer of the structure of the web site. Button icons also show the structure of the web site as well as provide access to other information sites. Images are detailed and tie into the overall theme of the site.

The overall structure of the page is repeated when the user scrolls to links at the bottom of the page. A consistent design and style-conservative and formal-is employed on subsequent pages (see Figure 2). Notice that the Wells Fargo name and page headers are repeated in the banners of each page, and that navigation buttons are provided to allow the viewer to quickly return to the initial page, send e-mail, conduct a search, or receive help.

Layering is obtained on this web page by using a hierarchy of headings that vary according to size and weight, as shown in Figure 3. Designers have grouped related text into brief "chunks" of information that can be read quickly. Information is further separated using horizontal and vertical lines, tables, bulleted lists, call-outs (in the form of a "Did you Know" checklist), and shading. Visual scan zones along the left side and bottom of the page enable viewers to quickly access information about other innovative products and services or locate other tools and information.

Small multiples or repeated shapes in the form of navigation buttons appear next to the point of entrance to a page (see Figure 3). The consistent shape, size, and placement of navigation buttons and icons makes them easy to find and use. In addition, viewers can focus on the detail within the icons themselves rather than on the shape of the icon. Although it is not apparent in the black and white figures of this publication, designers use bright color sparingly throughout the web page: blue text indicates hyperlinks, yellow adds perspective and dimension within detailed images, and yellow defines the edges of the call-out. The light background color scheme softens the page and makes the text easy to read. The text appears directly above, below, or to the side of images, and marquee text appears only on a small strip in the middle of the initial page.

In contrast to the Wells Fargo home page, the Volkswagen home page (http://www.vw.com, June 9,1997 ) is more lively and informal (see Figure 4). More importantly, the Volkswagen web page fails to incorporate Tufte's design principles.

Whereas the micro/macro structure of the Wells Fargo page was obvious, the micro/macro structure of the Volkswagen initial page and web site is less apparent. For example, although the Volkswagen logo appears, its use is incidental and the logo is nearly overwhelmed by the "Welcome" heading in the banner. In addition, the relationship between the images (a bicycle and a biker) and Volkswagen isn't immediately apparent, so viewers may be confused about the purpose of the page. Icons on the left side of the page are presented as headings and explained on the right side of the page; however, they provide no overall sense of the structure of the web site. In addition, a What's New hyperlink at the top of the page appears to be subordinate to the icons on the left of the page and is given equal or greater importance at the right side of the page.

Although the design and style of the Volkswagen web pages remain consistent-breezy and informal-with page headers appearing on each page, the banner "Volkswagen Trek" in Figure 5 gives no indication of how this individual page fits into the overall organization of the site. Thus, readers may have difficulty remembering where they are in relation to the initial home page. This problem is magnified because there are no navigation buttons on each page to return directly to the initial page.

Moreover, in Figure 5, the designers have separated information using a stationary frame on the left side and dotted vertical lines on the right. Unfortunately, this adds horizontal scrolling to vertical scrolling but doesn't layer the information. More important information is indicated solely by the use of bolding text within the center section and adding headings to the text at the right of the page.

Another problem with each page is that the icons have inconsistent shapes and sizes. The placement of the text around the icons is also inconsistent, sometimes appearing to the left and sometimes appearing to the right of the icon. Furthermore, although it is not apparent in the black and white figures of this publication, designers have used bright, bold colors throughout the web pages. Although this may appeal to the younger audience for which the site is intended, it can make the text difficult to read in the banners and left frame, and can cause a fuzzy edge around some images.

The Volkswagen site does contain several examples of effective design according to Tufte's principles. For example, detailed images of cars (see Figure 6) add interest and entice the viewer to examine the page further. Designers integrate visuals and text to create a feeling of motion and energy by rotating the image and wrapping text around it. In addition, short, choppy sentences and bolding of only part of a sentence, (see Figure 5) add to an overall sense of action and excitement. 


\section{Conclusion}

Edward Tufte may not have had web pages specifically in mind when he developed his principles for envisioning information, but a perusal of books written for web page designers clearly reveals Tufte's principles. In addition, Tufte's concepts of micro/macro design, layering and separation, small multiples. color and information, and integration of words and images provide a means of evaluating the effectiveness of the web pages in conveying information. Although this paper has examined only two web sites to illustrate Tufte's design principles, the World Wide Web offers a incxhaustible source of examples for teaching students and professionals about effective web page design.

\section{References}

Clark, C. L. (1996). Working the Web: A Student's Guide. Austin, TX: Harcourt Brace College Publishers.

Glover. J. M. (October 11, 1996).

http://www.glover.com/improve.html,).

Glover, J. M. (April 9, 1997).

http://www.glover.com/sucky.html,

Horton. W., Taylor, L., Ignacio, A., \& Hoft. N. L. (1996). The Web Page Design Cookbook: All the Ingredients You Nieed to Create 5-Star Web Pages. New York: John Wiley and Sons.

Morris, M.E. S., \& Hinrichs, R. J. (1996). Web Page Design: A Different Multimedia. Upper Saddle River. NJ: Prentice Hall.

Tilton, E., Steadman, C., \& Jones, T. (1996). Heb Weaving: Designing and Managing an Effective Web Site. Reading, MA: Addison-Wesley.

Tufte. E. R. (1983). The Visual Display of Quantutative Information. Cheshire, CT: Graphics Press.

Tufte, E. R. (1990). Envisioning Information. Cheshirc. CT: Graphics Press.

Volkswagen Home Page. (June 9. 1997). http://www.vw.com.

Wells Fargo Home Page. (June 9, 1997). http://www.wellsfargo.com. 


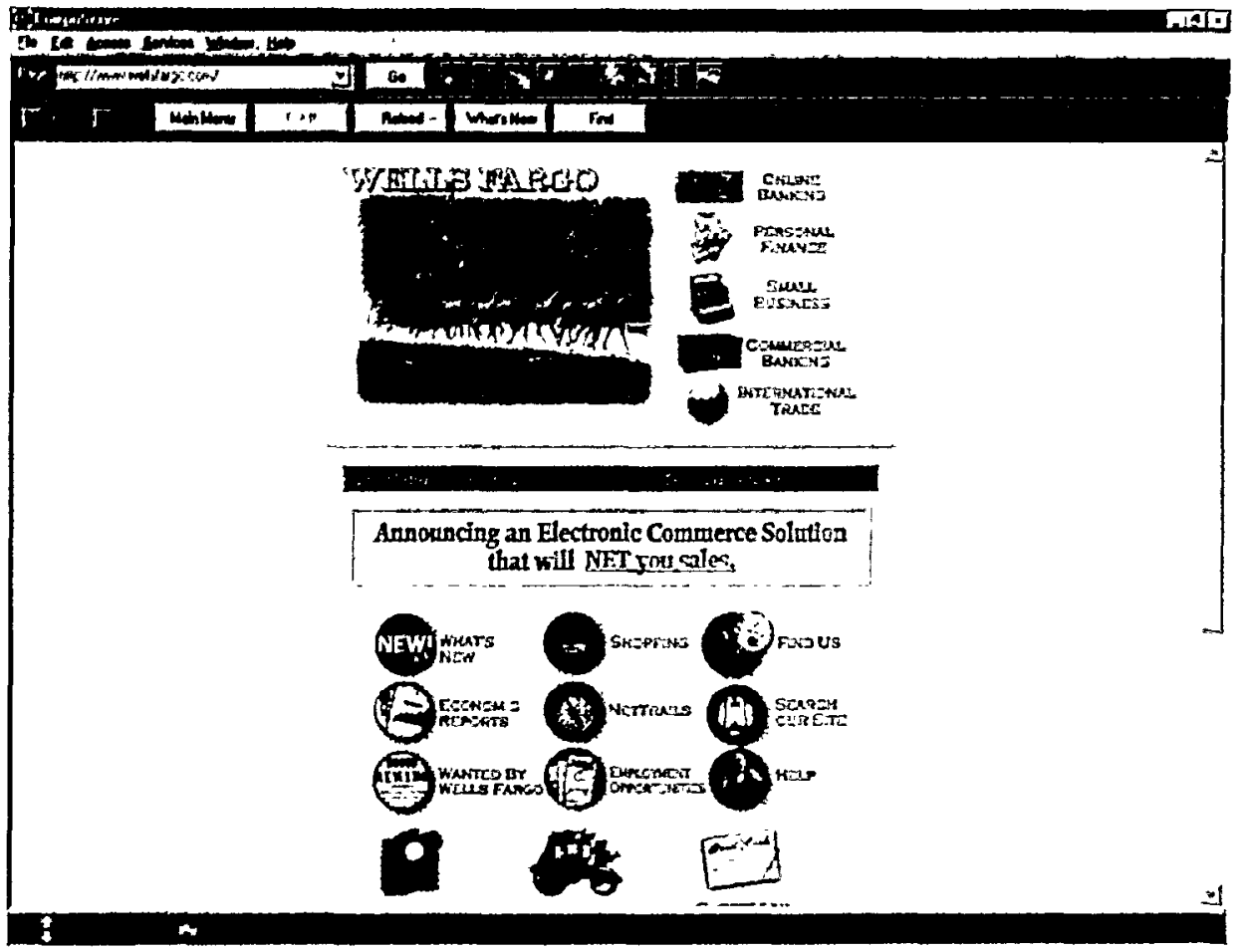

Figure 1. Wells Fargo Home Page.

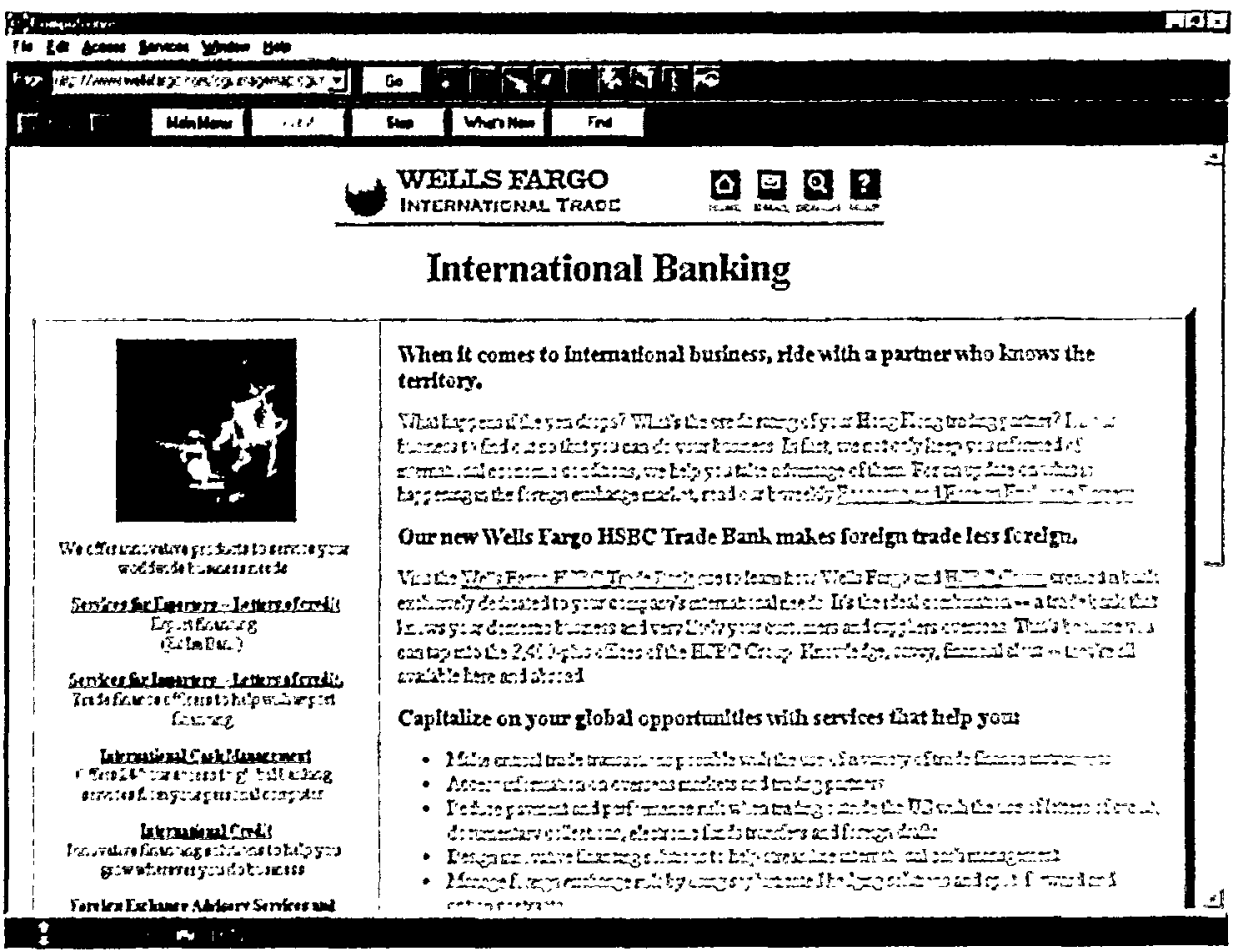

Figure 2. Web page design showing layering and separation of information and visual scan zones. 


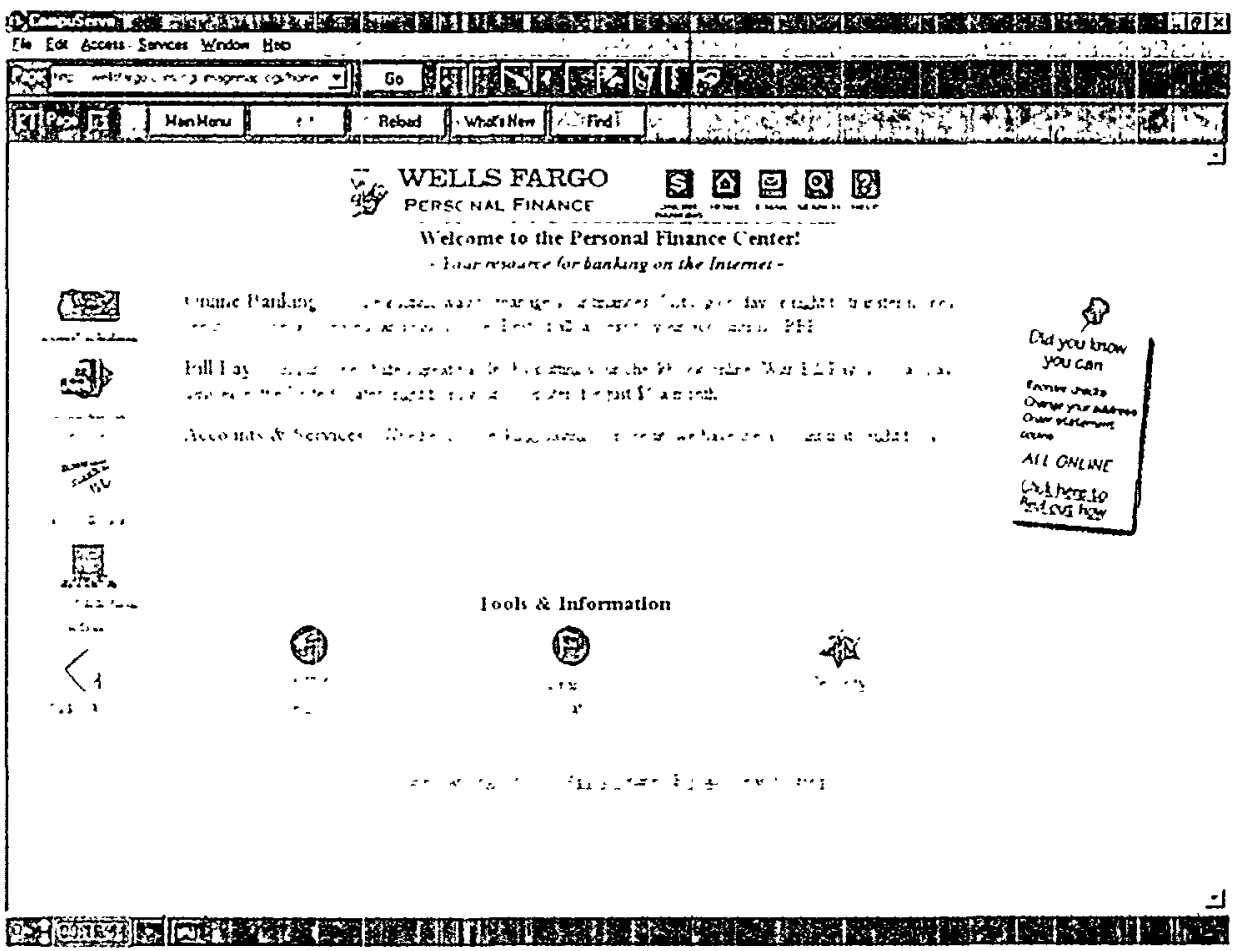

Figure 3. Web page design showing layering and separation of information and visual scan zones.

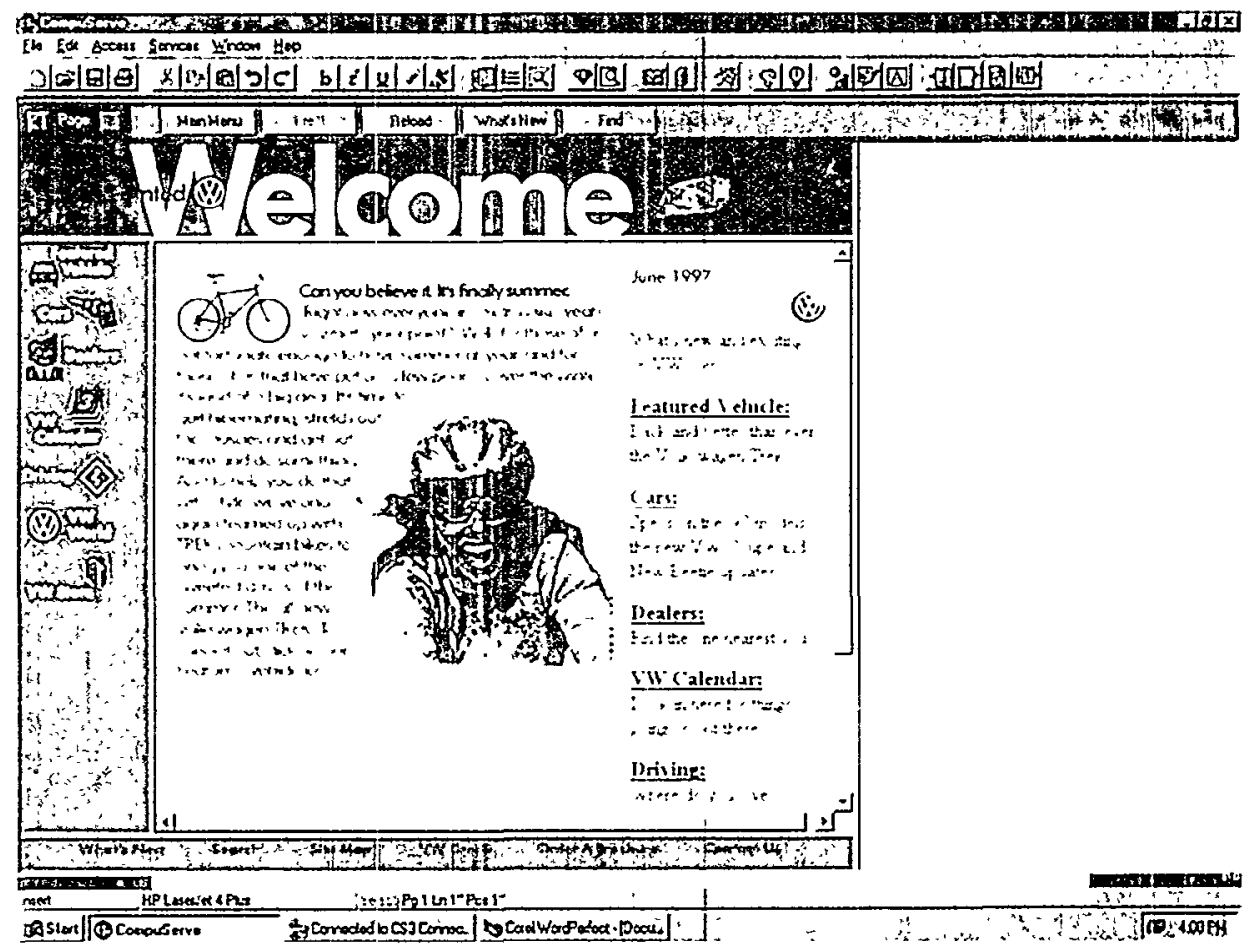

Figure 4. Volkswagen Home Page. 


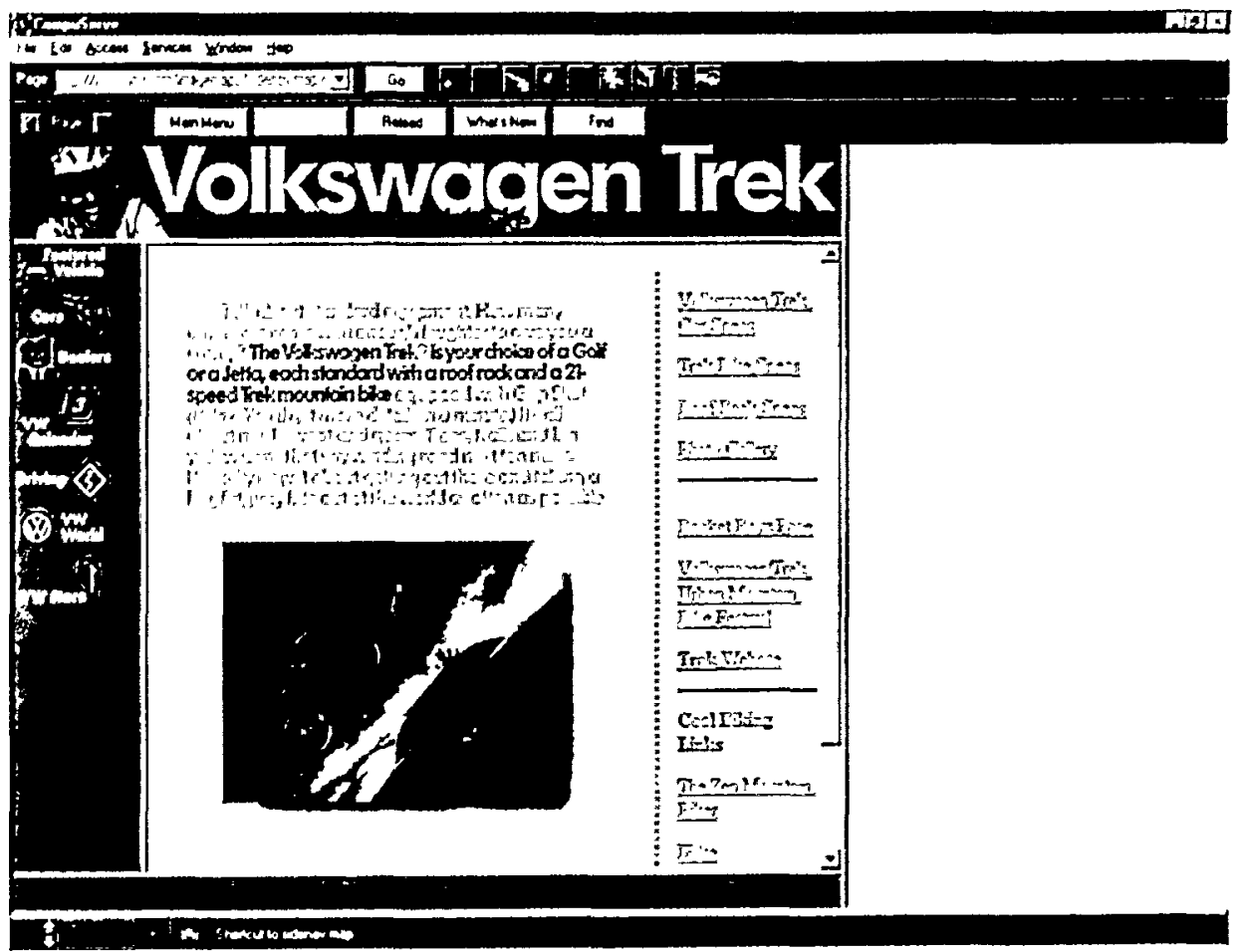

Figure 5. Web page with confusing macro/micro structure.

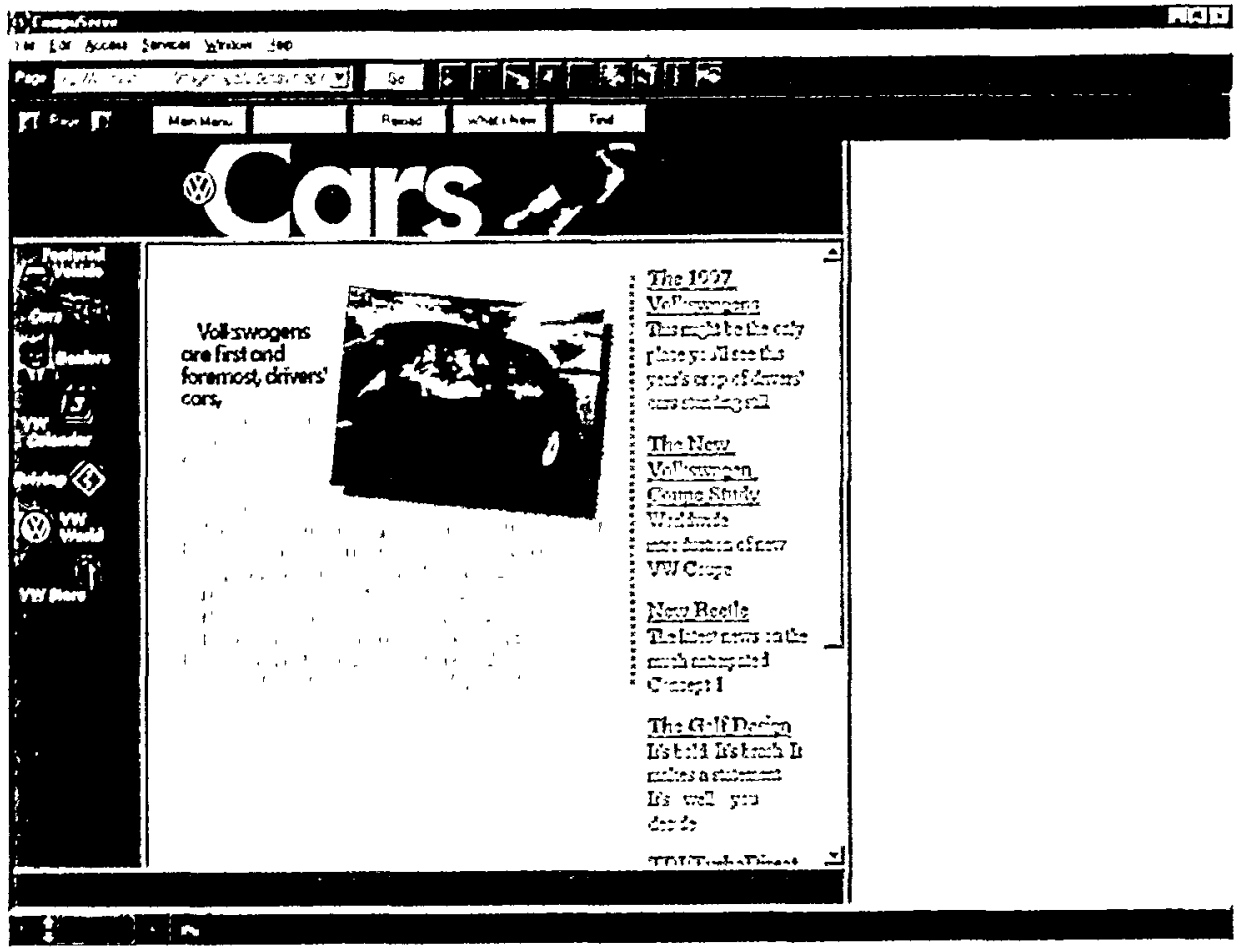

Figure 6. Web page with large areas of bold, bright colors. 